\title{
Amniotic membrane-assisted trabeculectomy for refractory glaucoma with corneal disorders
}

This article was published in the following Dove Press journal:

International Medical Case Reports Journal

25 January 2016

Number of times this article has been viewed

\section{Kazuhiko Mori \\ Yoko Ikeda \\ Yuko Maruyama \\ Shigeta Naruse \\ Morio Ueno \\ Shigeru Kinoshita}

Department of Ophthalmology, Kyoto Prefectural University of Medicine, Kyoto, Japan
Correspondence: Kazuhiko Mori

Department of Ophthalmology, Kyoto

Prefectural University of Medicine,

465 Kaji-cho, Hirokoji-agaru,

Kawaramachi-dori, Kamigyo-ku,

Kyoto 602-084I, Japan

Tel +81752515578

Fax $+8 \mid 7525 I 5663$

Email kmori@koto.kpu-m.ac.jp
Purpose: To report the cases of six consecutive patients who underwent amniotic membrane (AM)-assisted trabeculectomy (TLE) to treat refractory glaucoma with severe corneal disorders.

Methods: This study involved six patients (three males and three females, mean age: $69.5 \pm 15.8$ years) with refractory glaucoma and severe corneal disorders. The surgical procedure for each patient involved trabecular tissue being excised, and human AM then being placed epithelial side up on the corneal surface, sutured at the limbal sclera, and flipped over onto the sclera to cover the TLE area. The remaining edge of the AM was then inserted into the subconjunctival space and sutured. Medical records of all cases were reviewed with regard to intraocular pressure (IOP), visual acuity, and condition of the filtering bleb and ocular surface.

Results: The mean observation period was $69.5 \pm 15.8$ months, and mean IOP at presurgery and at 1,3 , and 7 years postoperative was $40.3 \pm 6.9,23.0 \pm 12.1,25.6 \pm 12.8$, and $28.5 \pm 19.1 \mathrm{mmHg}$, respectively. Glaucoma medications decreased from $3.0 \pm 1.1$ drugs (presurgery) to $0.8 \pm 1.0$ drugs (7 years postoperative). However, in some cases, ocular surface conditions or visual acuity worsened during the follow-up period.

Conclusion: Using AM as an internal patch for TLE, moderately good IOP control was obtained initially for the refractory glaucoma with severe corneal disorders; however, ocular surface conditions required special care, and the long-term IOP control was limited in some cases.

Keywords: amniotic membrane, refractory glaucoma, trabeculectomy

\section{Introduction}

Glaucoma with corneal disorders including ocular surface diseases is one of the most refractory types of glaucoma. Filtering surgery often fails due to severe inflammation, recurrent conjunctival scarring, proliferation of subconjunctival fibrous tissue, and the high risk of infection in patients taking immunosuppressive drugs. Other surgical procedures such as tube-shunt surgery are not always effective for severe ocular surface disorders because they may result in many problems such as cicatricial reaction of the conjunctivae, exposure of the tube or plate, and corneal endothelial damage.

Recently, the use of human amniotic membrane (AM) in ocular surface reconstruction has reportedly yielded good outcomes. ${ }^{1-7}$ In 1910, Davis ${ }^{8}$ first described the use of AM in skin transplantation procedures. In the field of ophthalmology, AM was reportedly used to repair conjunctival defects after chemical burns in the $1940 \mathrm{~s} ;{ }^{9,10}$ however, the results were not promising. Successful AM transplantation for ocular surface reconstruction was reported 50 years later, ${ }^{1,4}$ and AM is now the focus of attention as a 
biomaterial for the treatment of severe ocular surface diseases, such as ocular pemphigoid, Stevens-Johnson syndrome, and chemical burns. Koizumi et $\mathrm{al}^{6}$ and Nakamura et $\mathrm{al}^{7}$ reported very good prognoses when using AM as a transporter of cultured corneal epithelium to treat patients with severe ocular surface diseases. In ocular surface reconstruction, AM offers several benefits, as it can be easily obtained and preserved for several months, is not rejected immunologically, suppresses fibroblast proliferation, and accelerates cell migration and growth. Therefore, we theorized that AM might be suitable for glaucoma filtering surgery.

To date, there have been few reports on the use of AM in glaucoma surgery. ${ }^{11-14}$ Fujishima et $\mathrm{l}^{11}$ reported the effectiveness of AM for reducing intraocular pressure (IOP) in high-risk patients when it was placed under the scleral flap, yet Budenz et al ${ }^{12}$ found that AM transplantation was not an effective alternative to conjunctival advancement in patients undergoing the repair of leaking glaucoma filtering blebs; however, it should be noted that their total replacement of the leaking bleb with AM exposed it to constant pressure without supportive structures above or beneath the filtering bleb. In the presence of such structures, AM might be able to resist that pressure and function as part of the bleb wall. Based on these considerations, we theorized that an AM patch could be introduced below the filtering bleb. The purpose of this present study was to report six patients with refractory glaucoma having corneal severe disorders who were treated with AM-assisted trabeculectomy (TLE).

\section{Patients and methods}

This study involved six consecutive patients (three males and three females, mean age: $69.5 \pm 15.8$ years) with refractory glaucoma and corneal disorders who were treated with AM-assisted TLE between March 2000 and April 2002. Written informed consent was obtained from all patients for the use of AM, and the procedure was approved by the Human Studies Committee of Kyoto Prefectural University of Medicine. All experimental procedures were conducted in accordance with the tenets set forth in the Declaration of Helsinki. IOP measurements of each patient were obtained by use of a Goldmann applanation tonometer, and the surgical procedure for each patient was as follows. First, under local anesthesia, a limbal-based conjunctival flap was produced by careful detachment of the scarred conjunctiva, and an adequate amount of sclera was exposed. A limbal-based scleral flap was then produced and inoculated with a $0.4 \mathrm{mg} / \mathrm{mL}$ treatment of mitomycin C for 3 minutes, conventional TLE was performed, and the scleral flap was closed with 10-0 nylon

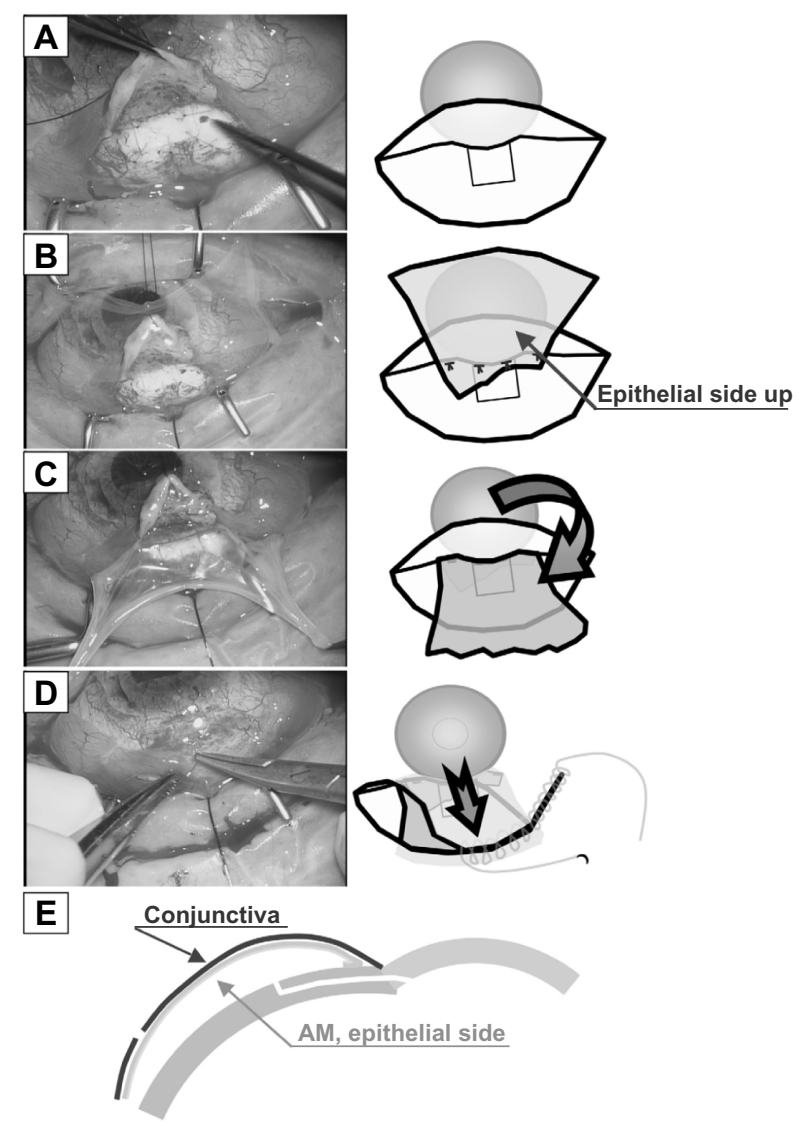

Figure I Images illustrating the new AM-assisted TLE procedure.

Notes: (A) First, conventional TLE with a limbus-based conjunctival flap and a 3-minute mitomycin C $(0.4 \mathrm{mg} / \mathrm{mL})$ treatment were performed. (B) AM, trimmed to the proper size, was then placed on the corneal surface with the epithelial side up. This was followed by suturing at the limbal sclera beside the scleral flap with 10-0 nylon sutures. (C) The other edge of the AM was then flipped over to cover the scleral flap. (D) Finally, a continuous conjunctival suturing with 10-0 polypropylene around the AM was performed. (E) A cross-sectional view of the filtering bleb with the AM patch. Abbreviations: AM, amniotic membrane; TLE, trabeculectomy.

sutures (Figure 1A). Next, deep-frozen human AM obtained at the time of cesarean section was thawed, trimmed to the proper size, placed epithelial side up on the corneal surface, and sutured at the limbal sclera beside the scleral flap using 10-0 nylon sutures (Figure 1B). The other edge of the AM was then flipped over (epithelial side down) to cover the scleral flap (Figure 1C). Finally, continuous conjunctival suturing with 10-0 polypropylene was performed (Figure 1D). A cross-sectional view of the filtering bleb with the AM patch is shown in Figure 1E.

The medical records of all six cases were reviewed in regard to each patient's IOP, visual acuity (VA), and the condition of the filtering bleb and ocular surface.

\section{Results}

The summary of all six cases is shown in Table 1, and the mean observation period was $69.5 \pm 15.8$ months. All cases 
had severe corneal disorders with secondary glaucoma, including pseudoexfoliation glaucoma, and five of the six cases had undergone penetrating keratoplasty (PKP). The details of three representative cases are described as follows. The first patient was a 49-year-old male with severe chemical burn who had undergone ocular surface reconstruction. Three months later, his IOP increased to $30 \mathrm{mmHg}$, and despite intensive medical therapy over a period of 6 months, it was judged that his eye required surgical treatment. Because of his severely scarred, adhesive subconjunctival tissue, we performed our newly developed surgical technique that consists of TLE with AM inserted under the AM that had been transplanted in the earlier procedure. Eight years after the AM-assisted TLE, his IOP was maintained at $20 \mathrm{mmHg}$ without any glaucoma medications. The second patient was a 58-year-old male with perforating corneal injury and traumatic cataract who had undergone intracapsular cataract extraction 40 years earlier. His eye progressed to secondary glaucoma, and he underwent several filtering surgeries to control IOP. Ten years before his current treatment, he had undergone PKP to treat bullous keratopathy due to corneal decompensation. However, his IOP became uncontrollable, and we performed AM-assisted TLE because of the conjunctival scarring caused by the previous surgical procedures. His mean IOP was maintained at $\sim 15.8 \mathrm{mmHg}$; however, due to the past severe damage, his VA became no light perception after several years. The third patient was a 57 -year-old female with a perforating corneal fungal infection. She had a previous history of perforating blunt trauma, resulting in lens prolapse. After undergoing PKP, her IOP increased up to $40 \mathrm{mmHg}$. The first TLE failed due to existing conjunctival scarring, and after stabilization of her conjunctival inflammation, she underwent AM-assisted TLE 8 months later.

In all six cases, filtering blebs, comprising an additional layer of AM with its epithelial side down, were established. The blebs functioned well without any leakage or wall thinning (Figure 2A-C). None of the patients experienced any ocular surface inflammation in the early postoperative phase, which often results in the failure of a conventional TLE in patients with glaucoma and ocular surface diseases.

The IOP time course of each case is shown in Figure 3. The mean IOP at presurgery and at 1,3 , and 7 years postoperative was $40.3 \pm 6.9,23.0 \pm 12.1,25.6 \pm 12.8$, and $28.5 \pm 19.1 \mathrm{mmHg}$, respectively. Glaucoma medications decreased from 3.0 \pm 1.1 drugs (presurgery) to $0.8 \pm 1.0$ (7 years postoperative). During the 7-year postoperative follow-up period, the IOP decreased compared with that at presurgery, although the patients' filtering blebs were

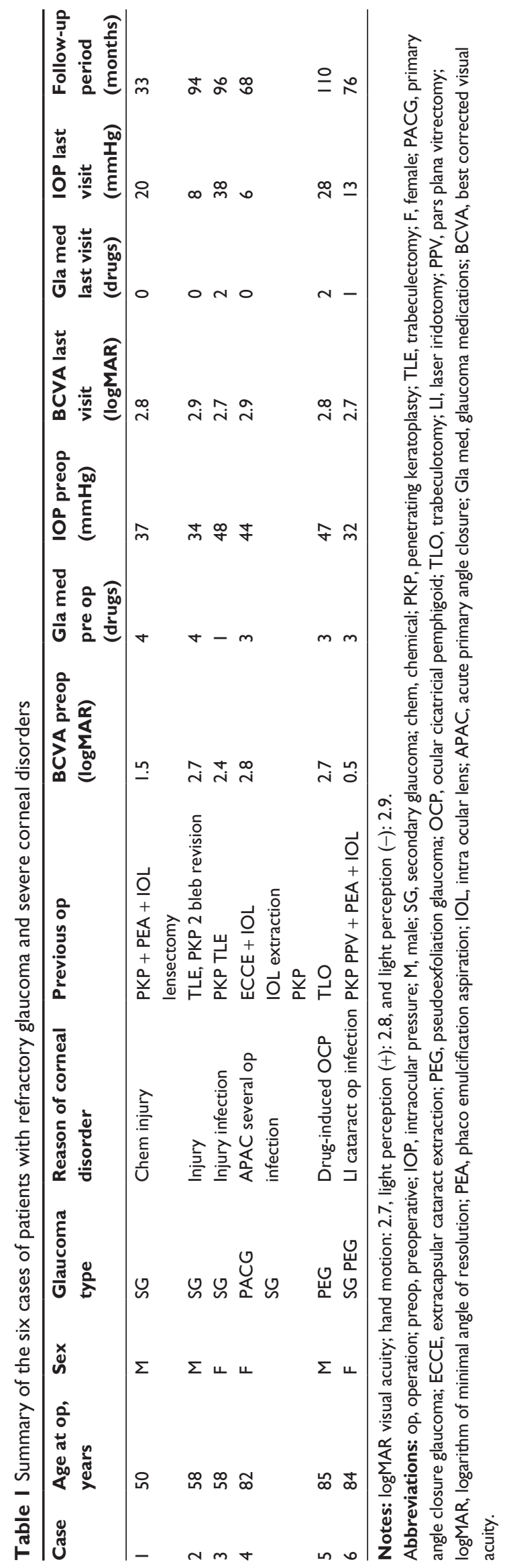




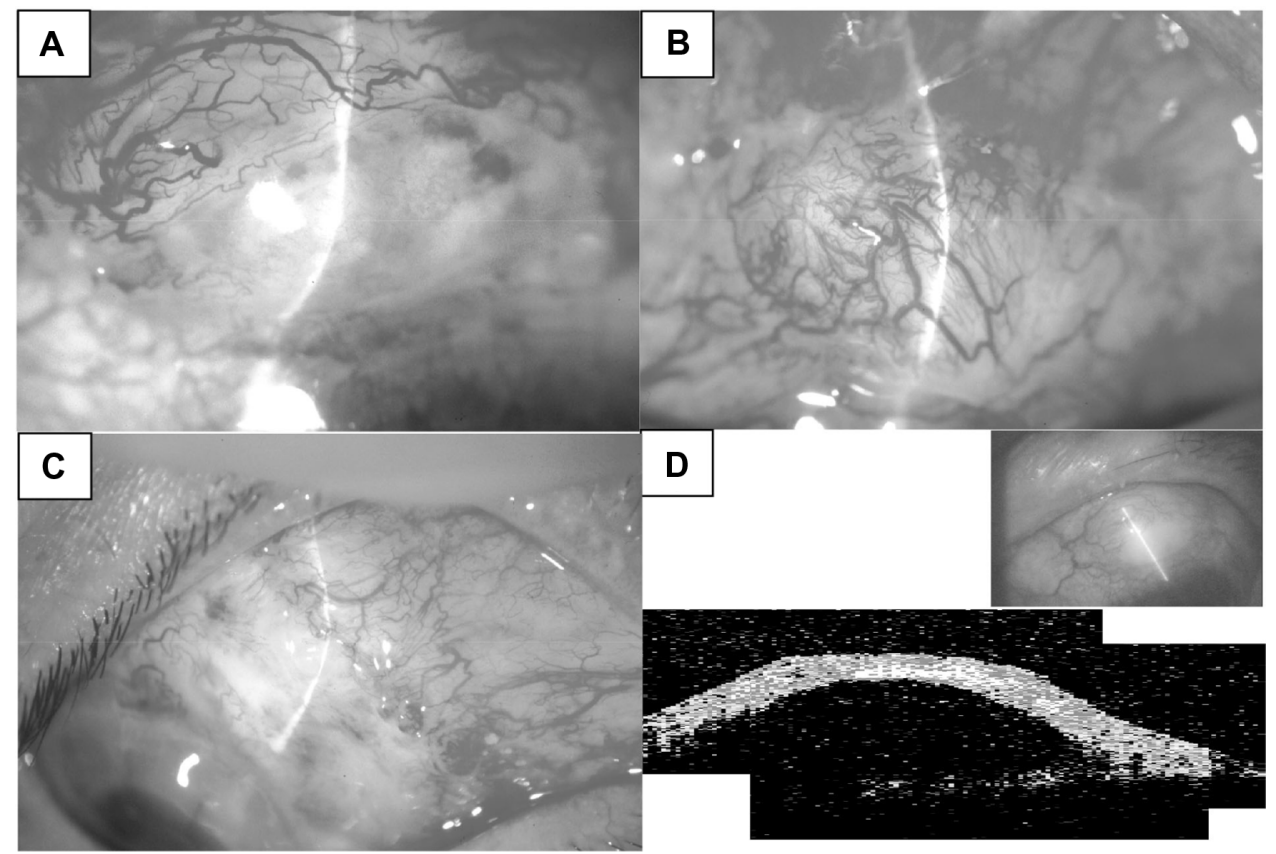

Figure 2 Images of three representative cases.

Notes: (A-C) Filtering blebs post AM-assisted TLE in all three patients. (D) Optical coherence tomography image (sagittal view) of the filtering bleb in case I at 2 years postoperative.

Abbreviations: AM, amniotic membrane; TLE, trabeculectomy.

gradually becoming small and flat. However, in some cases, the ocular surface conditions or VA worsened during the postoperative follow-up period.

We examined the subconjunctival structure of the filtering bleb in the first case using optical coherence tomography (OCT, OCT-3000; Carl Zeiss Meditec AG, Jena, Germany).
This is a noncontact, noninvasive method to visualize not only the bleb wall constructed by the AM but also the subconjunctival structure filled with aqueous humor. The OCT image showed a dome-shaped filtering bleb of equal thickness along the entire wall and a uniform subconjunctival lesion representing the aqueous humor posteriorly (Figure 2D).

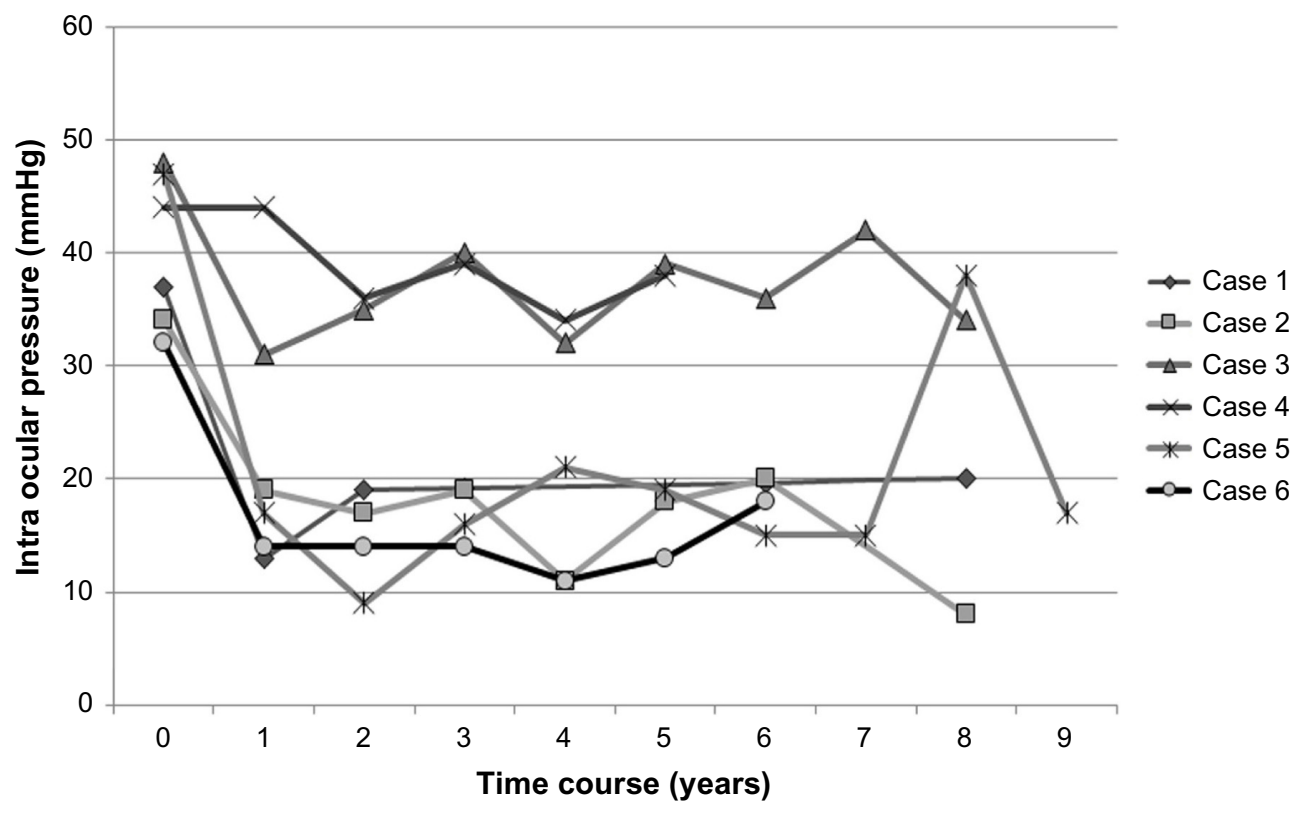

Figure 3 Time course of IOP changes in all six cases. Abbreviation: IOP, intraocular pressure. 


\section{Discussion}

The effects of subconjunctival AM transplantation are twofold, as the transplanted AM functions not only as a mechanical barrier but also as a biological modifier. As a mechanical barrier, it patches the conjunctival hole or tear, strengthens the fragile wall of the bleb, and prevents aqueous humor from overfiltrating and bacteria from entering into the subconjunctival and intraocular space. As a biological modifier, the AM offers a foundation and substrate for the conjunctival basal cells that stimulate epithelialization, ${ }^{15}$ traps polymorphonuclear cell infiltration, thereby preventing inflammation, ${ }^{16}$ promotes wound healing by inhibiting protease activity, ${ }^{17}$ and suppresses transforming growth factor (TGF)- $\beta$ signaling, thereby exerting antiscarring effects. $^{18}$

TGF- $\beta$ reportedly plays an important role in conjunctival scarring after glaucoma surgery. Cordeiro et $\mathrm{al}^{19}$ investigated the effects of TGF- $\beta 1,-\beta 2$, and $-\beta 3$ in vitro on Tenon's fibroblast contraction, proliferation, and migration. They concluded that TGF- $\beta$, which exists in the anterior chamber and subconjunctival space, is implicated in the conjunctival scarring of the filtering bleb. Their group also reported the effects of a new recombinant human antibody to TGF- $\beta 2$ on in vitro and in vivo conjunctival scarring and after glaucoma surgery. ${ }^{20,21}$ We expect subconjunctival AM transplantation to have effects similar to those elicited by the treatment of the subconjunctival tissue with anti-TGF- $\beta 2$ antibody.

The twofold effect of AM is derived from its histological structure, ie, the epithelial cellular monolayer and the stromal layer, which have different characteristics. The stromal layer is beneficial like an extracellular matrix that promotes cellular proliferation and migration on the conjunctival epithelium and epithelial cells produce cytokines to prevent severe scarring. Therefore, it may be important to place the AM epithelial side down on the scleral flap.

There are still many open questions regarding the use of AM in glaucoma surgery. For example, a question still exists as to whether or not the transplanted AM wall stays strong enough to maintain the filtering bleb wall for long periods. Also, it remains to be determined whether it is better to introduce the AM with the stromal side up or the epithelial side up on the scleral flap. In practice, the filtering bleb used for our first patient consisted of two layers of $\mathrm{AM}$ and lasted for $>2$ years without dissolving. To determine the long-term prognosis regarding IOP control by AM-assisted TLE, we continue to follow our patients carefully to identify and treat potential wound reopening or infection.
Recently, Nakamura et $\mathrm{al}^{22}$ also reported about AMassisted TLE, comparing the intrableb structures of AM-TLE with those of TLE alone using an ultrasound biomicroscope. The findings of that study revealed that the intrableb structures in AM-assisted TLE contained a wide fluid-filled space with a relatively thin wall, whereas those produced during TLE alone had no or minimal fluid-filled space. When the fluid-filled space extended posteriorly, IOP was reportedly well controlled, whereas if the eyes did not have any fluidfilled space or had a restricted space, the IOP control was poor. Case 1 in this study also showed a large fluid-filled space with good IOP control at 2 years postoperative when examined by OCT (Figure 2D), yet the bleb size gradually decreased and IOP increased as time passed.

\section{Conclusion}

Our new surgical technique, which uses AM as an internal patch for TLE, is an alternative treatment for patients with glaucoma and severe corneal disorders because of its initial good IOP control. However, special care and strict attention toward the condition of the ocular surface is required, and the long-term IOP control was limited in some cases.

\section{Acknowledgment}

The authors wish to thank John Bush for reviewing the manuscript.

\section{Disclosure}

The authors report no conflicts of interest in this work.

\section{References}

1. Kim JC, Tseng SC. Transplantation of preserved human amniotic membrane for surface reconstruction in severely damaged rabbit corneas. Cornea. 1995;14(5):473-484

2. Tsubota K, Satake Y, Ohyama M, et al. Surgical reconstruction of the ocular surface in advanced ocular cicatricial pemphigoid and StevensJohnson syndrome. Am J Ophthalmol. 1996;122(1):38-52.

3. Shimazaki J, Yang HY, Tsubota K. Amniotic membrane transplantation for ocular surface reconstruction in patients with chemical and thermal burns. Ophthalmology. 1997;104(12):2068-2076.

4. Tseng SC, Prabhasawat P, Lee SH. Amniotic membrane transplantation for conjunctival surface reconstruction. Am J Ophthalmol. 1997; 124(6):765-774.

5. Honavar SG, Bansal AK, Sangwan VS, et al. Amniotic membrane transplantation for ocular surface reconstruction in Stevens-Johnson syndrome. Ophthalmology. 2000;107(5):975-979.

6. Koizumi N, Inatomi T, Suzuki T, et al. Cultivated corneal epithelial stem cell transplantation in ocular surface disorders. Ophthalmology. 2001; 108(9):1569-1574

7. Nakamura T, Yoshitani M, Rigby H, et al. Sterilized, freeze-dried amniotic membrane: a useful substrate for ocular surface reconstruction. Invest Ophthalmol Vis Sci. 2004;45(1):93-99.

8. Davis JW. Skin transplantation with a review of 550 cases at the Johns Hopkins Hospital. Johns Hopkins Med J. 1910;15:307-396. 
9. de Rotth A. Plastic repair of conjunctival defects with fetal membrane. Arch Ophthalmol. 1940;23(3):522-525.

10. Sorsby A, Symmons HM. Amniotic membrane grafts in caustic burns of the eye (burns of second degree). Br J Ophthalmol. 1946;30(6): 337-345.

11. Fujishima H, Shimazaki J, Shinozaki N, et al. Trabeculectomy with the use of amniotic membrane for uncontrollable glaucoma. Ophthalmic Surg Lasers. 1998;29(5):428-431.

12. Budenz DL, Barton K, Tseng SC. Amniotic membrane transplantation for repair of leaking glaucoma filtering blebs. Am J Ophthalmol. 2000;130(5):580-588.

13. Barton K, Budenz DL, Khaw PT, et al. Glaucoma filtration surgery using amniotic membrane transplantation. Invest Ophthalmol Vis Sci. 2001;42(8):1762-1768.

14. Kee C, Hwang JM. Amniotic membrane graft for late-onset glaucoma filtering leaks. Am J Ophthalmol. 2002;133(6):834-835.

15. Shimazaki J, Shinozaki N, Tsubota K. Transplantation of amniotic membrane and limbal autograft for patients with recurrent pterygium associated with symblepharon. Br J Ophthalmol. 1998;82(3):235-240.

16. Park WC, Tseng SC. Modulation of acute inflammation and keratocyte death by suturing, blood, and amniotic membrane in PRK. Invest Ophthalmol Vis Sci. 2000;41(10):2906-2914.
17. Kim JS, Park SW, Kim JH, et al. Temporary amniotic membrane graft promotes healing and inhibits protease activity in corneal wound induced by alkali burn in rabbits. Invest Ophthalmol Vis Sci. 1998;39:S90.

18. Lee SB, Li DQ, Tan DT, et al. Suppression of TGF-beta signaling in both normal conjunctival fibroblasts and pterygial body fibroblasts by amniotic membrane. Curr Eye Res. 2000;20(4):325-334.

19. Cordeiro MF, Bhattacharya SS, Schultz GS, et al. TGF-beta1, beta2, and beta 3 in vitro: biphasic effects on Tenon's fibroblast contraction, proliferation, and migration. Invest Ophthalmol Vis Sci. 2000; 41(3):756-763.

20. Cordeiro MF, Gay JA, Khaw PT. Human anti-transforming growth factor-beta2 antibody: a new glaucoma anti-scarring agent. Invest Ophthalmol Vis Sci. 1999;40(10):2225-2234.

21. Siriwardena D, Khaw PT, King AJ, et al. Human antitransforming growth factor beta 2 monoclonal antibody - a new modulator of wound healing trabeculectomy: a randomized placebo controlled clinical study. Ophthalmology. 2002;109(3):427-431.

22. Nakamura M, Naka M, Tastumi Y, et al. Filtering bleb structure associated with long-term intraocular pressure control after amniotic membraneassisted trabeculectomy. Curr Eye Res. 2012;37(3):239-250.
International Medical Case Reports Journal

\section{Publish your work in this journal}

The International Medical Case Reports Journal is an international, peer-reviewed open-access journal publishing original case reports from all medical specialties. Previously unpublished medical posters are also accepted relating to any area of clinical or preclinical science. Submissions should not normally exceed 2,000 words or

\section{Dovepress}

4 published pages including figures, diagrams and references. The manuscript management system is completely online and includes a very quick and fair peer-review system, which is all easy to use. Visit $\mathrm{http}: / / \mathrm{www}$.dovepress.com/testimonials.php to read real quotes from published authors. 